\title{
Comportamento reológico e efeito da temperatura da polpa de pequi em diferentes concentrações
}

\author{
Rheological behavior and effect of temperature of pequi \\ pulp at different concentrations
}

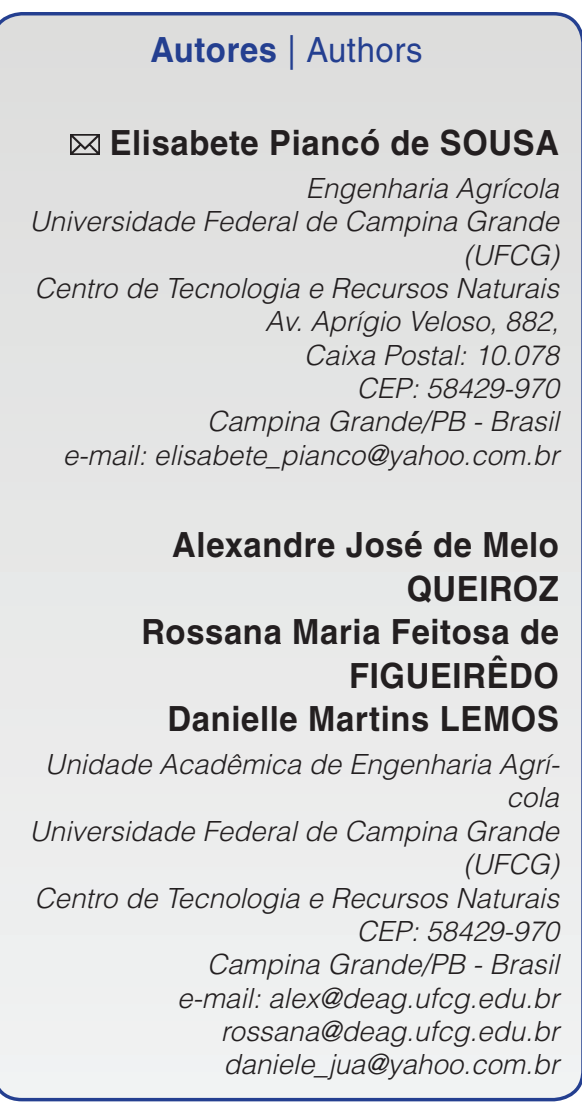

Autor Correspondente / Corresponding Author

Recebido: Abr. 16, 2014 Aprovado: Out. 14, 2014

\section{Resumo}

Apesar do elevado potencial econômico da polpa pequi ainda são escassos os trabalhos sobre suas propriedades físicas utilizadas em projetos e processos industriais. Diante disso, objetivou-se estudar o comportamento reológico da polpa de pequi com diferentes teores de sólidos solúveis totais $\left(6,8,10\right.$ e $12^{\circ}$ Brix) e em diferentes temperaturas $\left(25,30,35,40,45\right.$ e $\left.50^{\circ} \mathrm{C}\right)$. As medidas experimentais foram realizadas em viscosímetro Brookfield e os modelos reológicos de Ostwald-de-Waelle, Herschel-Bulkley e Mizrahi-Berk foram ajustados aos dados experimentais de tensão de cisalhamento e taxa de deformação. Dos modelos reológicos testados, os melhores ajustes foram observados empregando-se o modelo de Mizrahi-Berk. A polpa de pequi apresentou tendência de diminuição da viscosidade aparente com o aumento da taxa de deformação, comportando-se como fluido não newtoniano com características pseudoplásticas.

Palavras-chave: Caryocar coriaceum Wittm.; viscosidade; fluido não newtoniano.

\section{Summary}

Despite the high economic potential of pequi pulp, studies related to its physical properties for use in industrial projects and processes are still scarce. Therefore, this study aimed to assess the rheological behavior of pequi pulp with different total soluble solids contents $\left(6,8,10\right.$ and $12^{\circ}$ Brix) and at different temperatures $\left(25,30,35,40,45\right.$ and $\left.50^{\circ} \mathrm{C}\right)$. The experimental measurements were made using a Brookfield viscometer and the rheological models Ostwaldde-Waelle, Herschel-Bulkley and Mizrahi-Berk were fitted to the experimental data for shear stress and shear rate. Of the rheological models tested, the best fits were observed using the Mizrahi-Berk model. The pequi pulp showed a trend for decreasing apparent viscosity with increasing shear rate, behaving as a non-Newtonian fluid with pseudoplastic characteristics.

Key words: Caryocar coriaceum Wittm.; viscosity; non-Newtonian fluid. 


\section{Introdução}

Na parte mais setentrional do Nordeste brasileiro é encontrado o pequizeiro da espécie Caryocar coriaceum, que tem importante papel socioeconômico na Chapada do Araripe e circunvizinhança, nos estados do Ceará, Pernambuco e Piauí. Porém poucas são as informações disponíveis na literatura especializada dedicada a essa espécie, com reflexos negativos para a melhoria do sistema atual de exploração e, sobremaneira, para o surgimento de empreendimentos agroindustriais (OLIVEIRA et al., 2008).

Os frutos de pequi da espécie caryocar coriaceum são explorados para subsistência das famílias locais, sendo que, em função da produção sazonal, seu aproveitamento alimentar e econômico fica restrito a alguns meses do ano. Todavia, para permitir o uso da matéria-prima para a agroindústria, estudo de suas propriedades físicas, a fim de que os frutos possam ser beneficiados e comercializados durante o ano todo, mostra-se necessário

O conhecimento das propriedades reológicas de produtos alimentares é fundamental para os processos de concepção e avaliação de equipamentos, refletindo-se no projeto de tubulações, bombas, trocadores de calor, evaporadores, esterilizadores e misturadores. O estudo reológico é importante para identificar o comportamento do alimento sob diferentes condições de processamento (AHMED et al., 2005).

Segundo Oliveira et al. (2011), o comportamento reológico de sucos de frutas não pode ser descrito por uma equação newtoniana. Deste modo, desconsiderando sucos clarificados, é o padrão não newtoniano que costuma descrever adequadamente o comportamento de polpas, podendo esse ser identificado pelas curvas que descrevem os reogramas, representação gráfica da tensão de cisalhamento $(\tau)$ em função da taxa de deformação $(\dot{\gamma})$; ao contrário dos newtonianos, a viscosidade delas não é única, variando com a magnitude da taxa de cisalhamento.

Tendo em vista a falta de informações sobre as propriedades reológicas de frutos do Cerrado, dentre os quais o fruto do pequizeiro, neste trabalho objetivou-se estudar o comportamento reológico da polpa de pequi da espécie Caryocar coriaceum Wittm. com diferentes teores de sólidos solúveis totais e em diferentes temperaturas.

\section{Material e métodos}

\subsection{Processamento da matéria-prima}

A matéria-prima utilizada foi pequi (Caryocar coriaceum Wittm.) em estádio de maturação maduro, reconhecido quando a casca fica amolecida e o resquício do pedúnculo é removido com facilidade. Os frutos foram colhidos às seis horas, na safra de janeiro de 2011, na região da Chapada do Araripe, localizada no sul do estado do Ceará, e foram transportados para o laboratório em caixas de poliestireno expandido. No laboratório foram selecionados, mantendo-se apenas os maduros e intactos, sem nenhum dano físico. A seguir foram lavados em água corrente, sanitizados em solução de hipoclorito de sódio (100 ppm), enxaguados e despolpados por meio de uma despolpadeira marca Max Machine de aço inoxidável, que funciona com movimentos circulares e cuja parte interna, que mantém contato com o fruto, possui superfície abrasiva, a qual auxilia na extração da polpa. a polpa integral de pequi obtida foi embalada em sacos plásticos de polietileno de baixa densidade, os quais foram selados em seladora mecânica, e congelada em freezer a $-20^{\circ} \mathrm{C}$. A seguir, a polpa congelada foi colocada em caixas de poliestireno expandido e transportada em automóvel, no período noturno (sete horas de viagem) para a UFCG, onde chegou perfeitamente congelada, sem sinais de liquefação, sendo imediatamente armazenada em freezer até o momento das análises e ensaios aos quais foi submetida.

A polpa integral foi concentrada em evaporador rotativo da marca QUIMIS, modelo Q-344B2, sob vácuo, na temperatura de $60^{\circ} \mathrm{C}$. O teor de água inicial médio da polpa integral foi de $80 \%$ e o teor de sólidos solúveis totais, de 5 Brix. A partir desse valor definiu-se o teor de água das polpas concentradas utilizadas no estudo, que foram de 50, 60, 65 e 70\%, correspondentes, respectivamente, ao teor de sólidos solúveis totais de 12, 10, 8 e 6 'Brix. Foram realizadas análises de cinzas, lipídeos e proteínas conforme o Instituto Adolfo Lutz (IAL, 2008).

\subsection{Medidas reológicas}

A determinação do comportamento reológico das amostras concentradas (6, 8, 10 e $12^{\circ}$ Brix) foi feita em viscosímetro Brookfield DV-II+PRO provido de reservatório encamisado para pequenas amostras (determinador para pequenas amostras), aparelho que fornece leituras diretas de viscosidade aparente, tensão de cisalhamento e taxa de deformação, sem necessidade de conversões. A geometria do acessório é a de cilindros concêntricos, sendo que o cilindro interno, maciço, gira no interior do externo, em forma de copo. As leituras foram realizadas a $25,30,35,40,45$ e $50^{\circ} \mathrm{C}$, em taxas de deformação de 0,45 a 1,25 s $\mathrm{s}^{-1}$; o spindle utilizado foi o de número 29 e as leituras foram feitas depois de transcorridos os primeiros 30 segundos de cisalhamento.

A faixa de taxa de deformação utilizada nos experimentos para a polpa de pequi se encontra dentro da faixa $\left(10^{-3}\right.$ a $\left.1^{\circ}\right)$ presentes em processos de sedimentação, nivelamento de superfícies fluidas devido à tensão superficial, escoamento por gravidade, as quais são todas possíveis de ocorrer em embalagens, recipientes e processos de enchimento aplicáveis à polpa em estudo (STEFFE, 1996). 
Tabela 1. Modelos usados para determinar o comportamento reológico da polpa de pequi.

\begin{tabular}{|c|c|c|}
\hline Modelos & \multicolumn{2}{|c|}{ Equações } \\
\hline Ostwald-de-Waelle & $\tau=\mathrm{K} .(\dot{\gamma})^{\mathrm{n}}$ & (1) \\
\hline Herschel-Bulkley & $\tau-\tau_{0 H}=K_{H} \dot{\gamma}^{n_{H}}$ & (2) \\
\hline Mizrahi-Berk & $\tau^{0,5}=\mathrm{K}_{\mathrm{OM}}+\mathrm{K}_{\mathrm{M}}+\dot{\gamma}^{\mathrm{nM}}$ & (3) \\
\hline \multicolumn{3}{|c|}{ 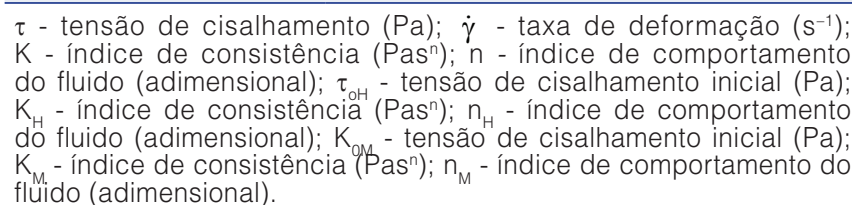 } \\
\hline
\end{tabular}

Os modelos reológicos de Ostwald-de-Waelle, Herschel-Bulkley e Mizrahi-Berk (Tabela 1) foram ajustados aos valores experimentais de tensão de cisalhamento em função da taxa de deformação de 0,45 a 1,25 s $\mathrm{s}^{-1}$, utilizando-se o programa Statistica versão 5.0.

Foram utilizados como critério de determinação de melhor ajuste dos modelos aos dados experimentais o coeficiente de determinação $\left(R^{2}\right)$ e o desvio percentual médio $(P)$, calculados conforme a Equação 4:

$$
P=\frac{100}{\eta} \sum_{t}^{n}=1\left|\frac{X_{\text {exp }} X_{\text {teor }}}{X_{\text {exp }}}\right|
$$

sendo: $P$ - desvio percentual médio (\%); $X_{\text {exp - valores }}$ obtidos experimentalmente; $X_{\text {teor }}$ - valores preditos pelo modelo; $\mathrm{n}$ - número de dados experimentais.

\subsection{Viscosidade aparente}

Foram traçadas as curvas da viscosidade aparente da polpa em função das taxas de deformação $(0,45$ a $1,25 \mathrm{~s}^{-1}$ ) utilizando-se os dados experimentais de viscosidade e valores teóricos calculados a partir do melhor modelo (Mizrahi-Berk).

\subsection{Efeito da temperatura}

Para a avaliação do efeito da temperatura sobre os valores da viscosidade aparente da polpa de pequi foi utilizada a equação de Arrhenius (Equação 5). O valor da viscosidade aparente ( $\mathrm{Pa}$ s) em função do inverso da temperatura $\left(\mathrm{K}^{-1}\right)$ correspondente à maior taxa de deformação $\left(1,25 \mathrm{~s}^{-1}\right)$ foi graficado com auxílio do programa computacional Origin 6.0, fazendo-se a regressão linear dos dados e obtendo-se os coeficientes da equação, a partir dos quais se calculou a energia de ativação.

$$
\eta=\eta_{\mathrm{o}} \exp \left[-\frac{\mathrm{Ea}}{\mathrm{RT}}\right]
$$

em que: $\eta$ - viscosidade aparente (Pa s); $\eta_{0}$ - viscosidade aparente inicial (Pa s); $\mathrm{T}$ - temperatura absoluta (K);
$\mathrm{R}$ - constante universal dos gases $\left(0,008314 \mathrm{~kJ} / \mathrm{mol} .{ }^{\circ} \mathrm{C}\right)$; Ea - energia de ativação para viscosidade $(\mathrm{kJ} / \mathrm{mol})$.

\subsection{Resultados e discussão}

Na polpa de pequi integral foi avaliada a composição centesimal, obtendo-se os seguintes resultados: teor de água $=80,73 \pm 0,14 \%$ (b.u.); cinzas $=0,16 \pm 0,02 \%$; lipídeos = 17,85 \pm 0,02\%; proteínas = 0,88 $\pm 0,12 \%$; e carboidratos totais $=0,38 \pm 0,01 \%$. Valores aproximados foram reportados por Arévalo-Pinedo et al. (2010) ao estudarem a composição centesimal da polpa de pequi da espécie Caryocar brasiliense.

\subsection{Ajuste dos modelos reológicos}

São apresentados na Tabela 2 os valores dos parâmetros do modelo reológico de Ostwald-de-Waelle, ajustados aos dados experimentais da curva de tensão de cisalhamento em função da taxa de deformação da polpa de pequi com diferentes teores de sólidos solúveis totais $\left(6,8,10\right.$ e $12^{\circ}$ Brix), a $25,30,35,40,45$ e $50^{\circ} \mathrm{C}$, os coeficientes de determinação $\left(R^{2}\right)$ e os desvios percentuais médios $(P)$.

A polpa de pequi apresentou altos coeficientes de determinação $\left(R^{2}\right)$, que foram acima de 0,82 , e valores de desvios percentuais médios $(P)$ inferiores a $10 \%$, os quais indicam que o modelo de Ostwald-de-Waelle pode ser utilizado para estimar os dados reológicos da polpa de pequi para a faixa de teor de sólidos solúveis totais nas temperaturas avaliadas. Guedes et al. (2010), estudando polpa de melancia, constataram que o modelo de Ostwaldde-Waelle pode ser utilizado satisfatoriamente para descrever o comportamento reológico em temperaturas de 10 a $60^{\circ} \mathrm{C}$ e em concentrações de $8,17,26$ e $35^{\circ}$ Brix, com $R^{2}$ superior a 0,974 . Ferreira et al. (2008) relataram, ao analisar as propriedades de escoamento da polpa de cupuaçu nas velocidades de rotação de 0,3 a 100 rpm e em temperaturas de 20 a $70^{\circ} \mathrm{C}$ que o modelo que melhor se ajustou aos dados experimentais foi o de Ostwald-deWaelle, o qual apresentou coeficiente de determinação $\left(R^{2}\right)$ superior a 0,94.

Os índices de consistência $(K)$ do modelo de Ostwald-de-Waelle aumentaram com o aumento dos sólidos solúveis totais da polpa, apresentando uma diminuição com a elevação da temperatura, de forma que os maiores valores de $\mathrm{K}$ foram obtidos na amostra mais concentrada ( $12^{\circ} \mathrm{Brix}$ ) e em temperaturas mais baixas, e os menores, na amostra menos concentrada e em temperaturas mais altas.

Do índice de comportamento do fluido (n), constata-se que todas as amostras apresentaram valores entre 0,2 e 0,3, confirmando a pseudoplasticidade delas em todas as concentrações e temperaturas. Ferreira et al. (2008), trabalhando com polpa de cupuaçu integral 
Tabela 2. Parâmetros do modelo de Ostwald-de-Waelle (Lei da potência), coeficientes de determinação ( $\left.R^{2}\right)$ e desvios percentuais médios $(\mathrm{P})$ para a polpa de pequi com diferentes teores de sólidos solúveis totais a diferentes temperaturas.

\begin{tabular}{|c|c|c|c|c|c|}
\hline \multirow{2}{*}{$\begin{array}{c}\text { Sólidos solúveis } \\
\text { totais ( }{ }^{\circ} \mathrm{Brix} \text { ) }\end{array}$} & \multirow[t]{2}{*}{ Temp. $\left({ }^{\circ} \mathbf{C}\right)$} & \multicolumn{2}{|c|}{ Parâmetros } & \multirow[t]{2}{*}{$\mathbf{R}^{2}$} & \multirow[t]{2}{*}{ P (\%) } \\
\hline & & K $\left(\right.$ Pas $\left.^{n}\right)$ & $\mathbf{n}$ & & \\
\hline \multirow{6}{*}{6} & 25 & 86,85 & 0,3524 & 0,9949 & 0,82 \\
\hline & 30 & 56,39 & 0,3301 & 0,9974 & 0,55 \\
\hline & 35 & 50,73 & 0,3245 & 0,9951 & 0,78 \\
\hline & 40 & 53,68 & 0,3184 & 0,9992 & 0,25 \\
\hline & 45 & 41,41 & 0,3388 & 0,9947 & 0,75 \\
\hline & 50 & 59,18 & 0,3088 & 0,9958 & 0,53 \\
\hline \multirow{6}{*}{8} & 25 & 145,38 & 0,3650 & 0,9914 & 1,14 \\
\hline & 30 & 88,11 & 0,3736 & 0,9955 & 0,85 \\
\hline & 35 & 78,16 & 0,3496 & 0,9953 & 0,79 \\
\hline & 40 & 67,25 & 0,3540 & 0,9970 & 0,63 \\
\hline & 45 & 84,87 & 0,3574 & 0,9980 & 0,58 \\
\hline & 50 & 74,24 & 0,3284 & 0,9974 & 0,57 \\
\hline \multirow{6}{*}{10} & 25 & 183,60 & 0,3449 & 0,9983 & 0,47 \\
\hline & 30 & 151,80 & 0,2994 & 0,9918 & 0,88 \\
\hline & 35 & 127,96 & 0,3665 & 0,9976 & 0,59 \\
\hline & 40 & 121,05 & 0,3454 & 0,9974 & 0,57 \\
\hline & 45 & 110,97 & 0,3550 & 0,9932 & 0,92 \\
\hline & 50 & 118,47 & 0,3306 & 0,9968 & 0,69 \\
\hline \multirow{6}{*}{12} & 25 & 217,30 & 0,3875 & 0,9983 & 0,53 \\
\hline & 30 & 174,02 & 0,2952 & 0,9979 & 0,43 \\
\hline & 35 & 126,77 & 0,3030 & 0,8881 & 3,45 \\
\hline & 40 & 116,32 & 0,2783 & 0,9940 & 0,71 \\
\hline & 45 & 112,85 & 0,3015 & 0,9956 & 0,68 \\
\hline & 50 & 119,05 & 0,2099 & 0,8267 & 3,03 \\
\hline
\end{tabular}

nas taxas de deformação de 0,3 a $300 \mathrm{~s}^{-1}$, encontraram valores de $n$ entre 0,23 e 0,42 em temperaturas de 10 a $60{ }^{\circ} \mathrm{C}$. Quando $\mathrm{n}=1$, o fluido é newtoniano, $\mathrm{n}$ diferente da unidade indica fisicamente o grau de desvio ou o afastamento do fluido do comportamento newtoniano, sendo que se $\mathrm{n}<1$ o comportamento é pseudoplástico e se $n>1$, é dilatante (TONELI et al., 2005).

Na Tabela 3 apresentam-se os parâmetros de ajuste do modelo de Herschel-Bulkley aos dados experimentais das amostras. O modelo é uma forma modificada do modelo proposto por Ostwald-de-Waelle.

O modelo de Herschel-Bulkley apresentou coeficientes de determinação $\left(R^{2}\right)$ acima de 0,93 e desvios percentuais médios $(P)$ inferiores a 10\%, sendo possível afirmar que o modelo prevê o comportamento reológico da polpa de pequi. Sharma et al. (1996), ao estudarem o comportamento reológico da polpa de tomate, observaram que o modelo de Herschel-Bulkley foi o mais adequado para predizer os dados da tensão de cisalhamento em função da taxa de deformação. Bezerra et al. (2009) constataram, estudando as propriedades reológicas da polpa de morango em temperaturas de 10 a $60^{\circ} \mathrm{C}$ e nas taxas de deformação de 0 a $300 \mathrm{~s}^{-1}$, que o melhor modelo foi o de Herschell-Bulkley, o qual apresentou os menores valores de erro experimental: de 0,08 a 2,67\%.

Observa-se que nas temperaturas avaliadas o valor de $\left(\tau_{\mathrm{OH}}\right)$ aumentou com o aumento dos sólidos solúveis totais. A tensão de cisalhamento inicial $\left(\tau_{O H}\right)$ é a tensão mínima necessária para o fluido começar a escoar, significando que quanto maior o teor de sólidos solúveis totais da polpa de pequi maior será $\tau_{0 \mathrm{H}}$, e como a viscosidade diminui com o aumento da temperatura, $\tau_{0 H}$ será menor. De maneira geral, em uma mesma temperatura, com o aumento dos sólidos solúveis totais houve tendência de aumento do índice de consistência $\left(\mathrm{K}_{\mathrm{H}}\right)$; na polpa estudada, o maior índice de consistência $\left(K_{H}\right)$ foi de 166,50 Pas ${ }^{n}$ e o menor, de 14,28 Pas ${ }^{n}$.

$\mathrm{O}$ índice de comportamento do fluido $\left(\mathrm{n}_{H}\right)$ não apresentou tendência definida em relação ao aumento da temperatura e do teor de sólidos solúveis totais. Augusto et al. (2012), estudando as propriedades reológicas da polpa de seriguela em temperaturas de 0, 20, 40, 60 e $80^{\circ} \mathrm{C}$, em taxas de deformação variando de 0 a $100 \mathrm{~s}^{-1}$, obtiveram bons ajustes pelo modelo de Herschel-Bulkley, com o índice de comportamento do fluido variando entre 0,25 e 0,48. 
Tabela 3. Parâmetros do modelo de Herschel-Bulkley, coeficientes de determinação $\left(R^{2}\right)$ e desvios percentuais médios $(P)$ para a polpa de pequi com diferentes teores de sólidos solúveis totais e em diferentes temperaturas.

\begin{tabular}{|c|c|c|c|c|c|c|}
\hline \multirow{2}{*}{$\begin{array}{l}\text { Sólidos solúveis } \\
\text { totais ( }{ }^{\circ} \mathrm{Brix} \text { ) }\end{array}$} & \multirow[t]{2}{*}{ Temp. $\left({ }^{\circ} \mathbf{C}\right)$} & \multicolumn{3}{|c|}{ Parâmetros } & \multirow[t]{2}{*}{$\mathbf{R}^{2}$} & \multirow[t]{2}{*}{ P (\%) } \\
\hline & & $\tau_{\text {оH }}(\mathbf{P a})$ & $\mathrm{K}_{\mathrm{H}}\left(\operatorname{Pas}^{n}\right)$ & $\mathbf{n}_{\mathrm{H}}$ & & \\
\hline \multirow{6}{*}{6} & 25 & 45,26 & 41,10 & 0,8346 & 0,9995 & 0,25 \\
\hline & 30 & 25,48 & 30,72 & 0,6565 & 0,9996 & 0,23 \\
\hline & 35 & 27,40 & 23,09 & 0,7990 & 0,9996 & 0,18 \\
\hline & 40 & 17,46 & 36,13 & 0,4945 & 0,9998 & 0,11 \\
\hline & 45 & 22,04 & 19,15 & 0,8220 & 0,9994 & 0,27 \\
\hline & 50 & 30,37 & 28,60 & 0,7042 & 0,9989 & 0,29 \\
\hline \multirow{6}{*}{8} & 25 & 31,35 & 113,83 & 0,4794 & 0,9917 & 1,08 \\
\hline & 30 & 43,77 & 43,84 & 0,8375 & 0,9998 & 0,13 \\
\hline & 35 & 40,67 & 37,06 & 0,8254 & 0,9998 & 0,15 \\
\hline & 40 & 31,34 & 35,62 & 0,7316 & 0,9998 & 0,11 \\
\hline & 45 & 34,86 & 49,72 & 0,6560 & 0,9997 & 0,16 \\
\hline & 50 & 34,98 & 38,98 & 0,6818 & 0,9999 & 0,08 \\
\hline \multirow{6}{*}{10} & 25 & 75,35 & 107,68 & 0,6306 & 0,9999 & 0,09 \\
\hline & 30 & 92,51 & 58,40 & 0,8976 & 0,9991 & 0,28 \\
\hline & 35 & 55,28 & 72,17 & 0,7050 & 0,9999 & 0,10 \\
\hline & 40 & 54,54 & 66,05 & 0,6880 & 0,9997 & 0,17 \\
\hline & 45 & 61,38 & 48,85 & 0,9200 & 0,9993 & 0,29 \\
\hline & 50 & 34,70 & 83,58 & 0,4874 & 0,9973 & 0,59 \\
\hline \multirow{6}{*}{12} & 25 & 80,82 & 135,74 & 0,6628 & 0,9998 & 0,14 \\
\hline & 30 & 7,50 & 166,50 & 0,3097 & 0,9979 & 0,44 \\
\hline & 35 & 101,70 & 21,05 & 2,8404 & 0,9900 & 0,84 \\
\hline & 40 & 7,48 & 108,82 & 0,3082 & 0,9928 & 0,80 \\
\hline & 45 & 40,50 & 72,16 & 0,4953 & 0,9964 & 0,60 \\
\hline & 50 & 102,11 & 14,28 & 2,8148 & 0,9345 & 1,93 \\
\hline
\end{tabular}

De acordo com a Tabela 4, onde são apresentados os parâmetros de ajuste para o modelo de Mizrahi-Berk, verifica-se que os coeficientes de determinação $\left(R^{2}\right)$ foram superiores aos encontrados pelos demais modelos, sendo que na maioria das amostras situou-se em torno de 0,99. Os desvios percentuais médios também se mantiveram reduzidos, apresentando valores no intervalo de 0,04 a $0,72 \%$. Com esses resultados pode-se afirmar que o modelo de Mizrahi-Berk foi o que melhor descreveu o comportamento da polpa de pequi nas concentrações e temperaturas utilizadas.

Existem na literatura alguns trabalhos com polpa de frutas que verificaram em testes com diversos modelos que o de Mizrahi-Berk ajustou-se melhor ao comportamento reológico, a exemplo de Fernandes et al. (2008), que trabalharam com polpa de umbu-cajá em diferentes temperaturas $\left(10\right.$ a $\left.50{ }^{\circ} \mathrm{C}\right)$ e velocidades de rotação $(0,5 ; 1,0 ; 2,5 ; 10 ; 20 ; 50$ e 100 rpm), obtendo coeficiente de determinação $\left(R^{2}\right)$ superior a 0,98 e erros percentuais médios $(P)$ menores que $2,5 \%$.

Verifica-se que com o aumento da temperatura o parâmetro $K_{0 M}$ não apresentou tendência definida e que com o aumento dos sólidos solúveis totais ocorre uma tendência de aumento: os valores variaram entre
1,62 e 10,09 Pa s; o índice de consistência $\left(K_{M}\right)$ das amostras com teores de sólidos solúveis totais de 6,8 e $10{ }^{\circ}$ Brix foram próximos; o parâmetro $n_{M}$ do modelo, que corresponde ao índice de comportamento do fluido, para as amostras com 6,8 e $10^{\circ}$ Brix, foram inferiores a 1 , resultado comum para a maioria das polpas.

Na Figura 1 tem-se os gráficos dos valores da tensão de cisalhamento em função da taxa de deformação para as amostras de pequi com diferentes teores de sólidos solúveis totais, nas temperaturas de 25 a $50{ }^{\circ} \mathrm{C}$, com ajustes pelo modelo de Mizrahi-Berk. Observa-se em todas as concentrações que às temperaturas mais baixas correspondem os maiores valores de viscosidade, denotado pela posição mais alta das curvas na relação tensão de cisalhamento e taxa de deformação. Esse efeito da temperatura é mais evidente nas amostras com os maiores teores de sólidos solúveis totais (10 e $12{ }^{\circ} \mathrm{Brix}$ ), isso ficou demonstrado visualmente com os resultados constatados nas energias de ativação. Da mesma forma, verifica-se pelo cruzamento entre curvas das diferentes temperaturas que, à medida que as polpas têm diminuídos os sólidos solúveis totais, a diferença de $5{ }^{\circ} \mathrm{C}$ entre temperaturas subsequentes tem pouca influência na posição das curvas. 
Tabela 4. Parâmetros do modelo de Mizrahi-Berk, coeficientes de determinação $\left(R^{2}\right)$ e desvios percentuais médios $(P)$ para a polpa de pequi com diferentes teores de sólidos solúveis totais e em diferentes temperaturas.

\begin{tabular}{|c|c|c|c|c|c|c|}
\hline \multirow{2}{*}{$\begin{array}{l}\text { Sólidos solúveis } \\
\text { totais ( }{ }^{\circ} \text { Brix) }\end{array}$} & \multirow[t]{2}{*}{ Temp. $\left({ }^{\circ} \mathbf{C}\right)$} & \multicolumn{3}{|c|}{ Parâmetros } & \multirow[t]{2}{*}{$\mathbf{R}^{2}$} & \multirow[t]{2}{*}{ P (\%) } \\
\hline & & $\mathrm{K}_{\text {ом }}(\mathrm{Pa})$ & $K_{M}\left(\operatorname{Pas}^{n}\right)$ & $\mathbf{n}_{\mathrm{m}}$ & & \\
\hline \multirow{6}{*}{6} & 25 & 6,51 & 2,78 & 0,6647 & 0,9995 & 0,13 \\
\hline & 30 & 4,79 & 2,70 & 0,4984 & 0,9995 & 0,12 \\
\hline & 35 & 5,08 & 2,01 & 0,6443 & 0,9996 & 0,10 \\
\hline & 40 & 3,65 & 3,66 & 0,3326 & 0,9998 & 0,05 \\
\hline & 45 & 4,55 & 1,86 & 0,6599 & 0,9994 & 0,14 \\
\hline & 50 & 5,30 & 2,37 & 0,5518 & 0,9988 & 0,15 \\
\hline \multirow{6}{*}{8} & 25 & 5,34 & 6,70 & 0,3420 & 0,9917 & 0,53 \\
\hline & 30 & 6,37 & 2,99 & 0,6563 & 0,9998 & 0,07 \\
\hline & 35 & 6,14 & 2,66 & 0,6500 & 0,9998 & 0,07 \\
\hline & 40 & 5,32 & 2,85 & 0,5578 & 0,9998 & 0,06 \\
\hline & 45 & 5,50 & 3,70 & 0,4797 & 0,9997 & 0,08 \\
\hline & 50 & 5,62 & 2,97 & 0,5202 & 0,9999 & 0,04 \\
\hline \multirow{6}{*}{10} & 25 & 8,03 & 5,50 & 0,4567 & 0,9999 & 0,05 \\
\hline & 30 & 9,46 & 2,81 & 0,7600 & 0,9990 & 0,15 \\
\hline & 35 & 6,70 & 4,30 & 0,5256 & 0,9999 & 0,05 \\
\hline & 40 & 7,01 & 3,96 & 0,5228 & 0,9997 & 0,09 \\
\hline & 45 & 7,60 & 2,90 & 0,7361 & 0,9993 & 0,14 \\
\hline & 50 & 5,14 & 5,73 & 0,3271 & 0,9971 & 0,29 \\
\hline \multirow{6}{*}{12} & 25 & 8,27 & 6,44 & 0,4751 & 0,9998 & 0,07 \\
\hline & 30 & 1,62 & 11,56 & 0,1694 & 0,9978 & 0,16 \\
\hline & 35 & 10,07 & 1,00 & 2,6631 & 0,9883 & 0,42 \\
\hline & 40 & 2,75 & 8,03 & 0,1898 & 0,9933 & 0,36 \\
\hline & 45 & 6,01 & 4,60 & 0,3683 & 0,9962 & 0,30 \\
\hline & 50 & 10,09 & 0,68 & 2,6928 & 0,9390 & 0,72 \\
\hline
\end{tabular}

\subsection{Comportamento da viscosidade aparente}

Na Figura 2 encontram-se as viscosidades aparentes experimentais e teóricas, calculadas a partir do modelo de Mizrahi-Berk, da polpa concentrada de pequi com teores de sólidos solúveis totais de 6, 8, 10 e 12 Brix, nas taxas de deformação de 0,45; 0,5;0,625;0,75; 1,0 e $1,25 \mathrm{~s}^{-1}$ e nas temperaturas $25,30,35,40,45$, e $50{ }^{\circ} \mathrm{C}$. Observou-se que em todas as amostras as viscosidades aparentes experimental e teórica apresentaram tendência de diminuição com o aumento da taxa de deformação, em toda a faixa de temperatura estudada, tornando-se mais fluidas, comportamento característico de fluidos pseudoplásticos. Assim como a viscosidade aparente também diminuíu com o aumento do teor de sólidos solúveis totais. Ainda de acordo com a Figura 2, pode-se afirmar que a polpa concentrada de pequi apresentou valores de viscosidade aparente na faixa de 60,00 a 375,00 Pa s.

Vale ressaltar que na polpa com teor de sólidos solúveis totais de 10 e $12{ }^{\circ}$ Brix verificou-se diminuição da viscosidade com o aumento da temperatura entre 25 e $45^{\circ} \mathrm{C}$, e aumento da viscosidade com o aumento entre 45 e $50^{\circ} \mathrm{C}$. A polpa com $8^{\circ}$ Brix teve diminuição na viscosidade aparente com o aumento da temperatura entre 25 e $40^{\circ} \mathrm{C}$ e entre as temperaturas de 45 e $50{ }^{\circ} \mathrm{C}$. Na polpa com $6{ }^{\circ}$ Brix, a redução da viscosidade foi entre as temperaturas de 25,30 e $35^{\circ} \mathrm{C}$.

Dentre trabalhos que citam valores que se aproximam dos encontrados neste estudo, os que mais se assemelham são os de Oliveira et al. (2011), que relataram viscosidades aparentes para polpa de goiaba, a $35^{\circ} \mathrm{C}$, entre 400 e 200 Pa s, para taxas de deformação entre 0 e $10 \mathrm{~s}^{-1}$. Com relação ao comportamento pseudoplástico apresentado na polpa estudada, foi também verificado por Oliveira et al. (2012) para a polpa de morango com $6,80^{\circ}$ Brix, nas temperaturas $20,25,30$ e $35^{\circ} \mathrm{C}$ e em taxas de deformação entre 1 a $16 \mathrm{~s}^{-1}$; e por Vidal et al. (2006), para a polpa de manga centrifugada, nas temperaturas 10 e $60^{\circ} \mathrm{C}$ e em taxas de deformação variando de 0 a $300 \mathrm{~s}^{-1}$.

Trabalhos com resultados semelhantes em relação a faixa de viscosidade e a taxa de deformação foram feitos por: Oliveira et al. (2011), que relataram viscosidade aparente para polpa de goiaba, a $35^{\circ} \mathrm{C}$, entre 400 e $200 \mathrm{~Pa} \mathrm{~s}$, para taxas de deformação entre 0 e $10 \mathrm{~s}^{-1}$; por Vriesmann et al. (2009), para polpa de cupuaçu com amido (6\%), com viscosidades de $100 \mathrm{~Pa} \mathrm{~s}$ a $10 \mathrm{~Pa} \mathrm{~s}$, 

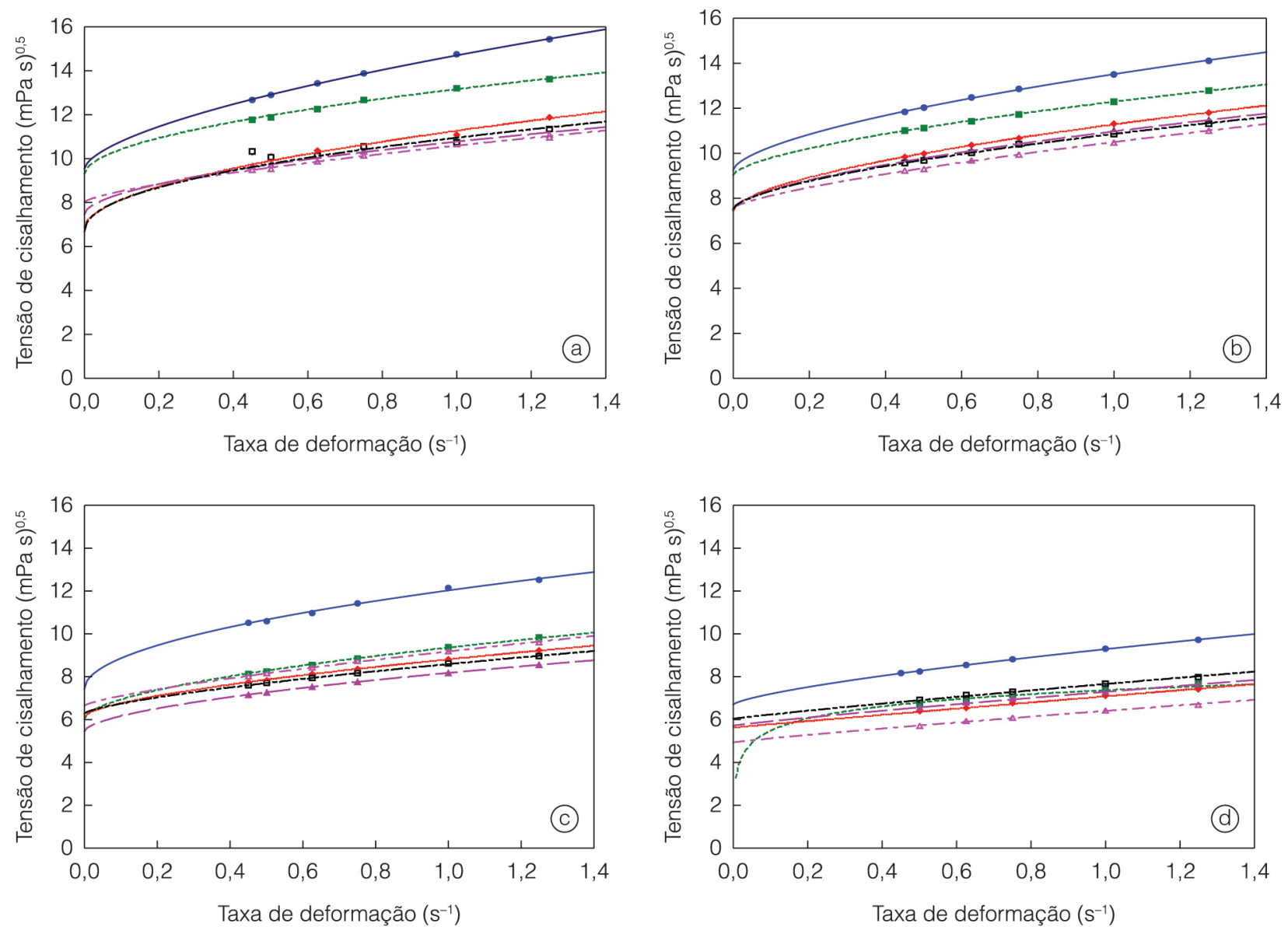

- $25^{\circ} \mathrm{C}-30^{\circ} \mathrm{C} \cdot 35^{\circ} \mathrm{C}$

$\triangle 40^{\circ} \mathrm{C} \triangle 45^{\circ} \mathrm{C} \quad \square 50^{\circ} \mathrm{C}$

Figura 1. Relação da tensão de cisalhamento em função da taxa de deformação para a polpa de pequi com diferentes teores de sólidos solúveis totais, com ajustes pelo modelo de Mizrahi-Berk: (a) $12^{\circ} \mathrm{Brix}$; (b) $10^{\circ} \mathrm{Brix}$; (c) $8{ }^{\circ} \mathrm{Brix}$; (d) $6{ }^{\circ} \mathrm{Brix}$.

para taxas de deformação na faixa de 0,1 a $1 \mathrm{~s}^{-1}$; e por Izidoro et al. (2008), para formulações elaboradas com a polpa de banana verde com incorporação de emulsões de óleo e água, as quais apresentaram valores entre 5 e 6 Pa s para taxas de deformação entre 0 e $25 \mathrm{~s}^{-1}$.

\subsection{Energia de ativação}

$\mathrm{Na}$ Tabela 5 apresentam-se os valores de energia de ativação $\left(E_{a}\right)$ das amostras concentradas de pequi.

Os valores de $\mathrm{R}^{2}$ iguais ou maiores que 0,94, para todas as amostras, indicam bom ajuste da equação de Arrhenius. As energias de ativação mostraram tendência de redução com o aumento dos sólidos solúveis totais. Observou-se que as menores concentrações correspondem às menores viscosidades, tais resultados corroboram os verificados por Sato e Cunha (2007) em seu estudo da energia de ativação em polpa de jaboticaba, no qual relatam que o material com viscosidade baixa apresentou maior energia de ativação, enquanto que em amostras com viscosidade mais elevada a energia de ativação tendeu a diminuir. O mesmo foi observado por Chin et al. (2009) em estudo do comportamento reológico do suco de pomelo em temperaturas de 6 a $75^{\circ} \mathrm{C}$ e concentração de sólidos solúveis de 20 , 30 e $50^{\circ}$ Brix, no qual verificaram valores de energia de ativação de $34,02 \mathrm{~kJ} / \mathrm{mol}$ (20 ${ }^{\circ}$ Brix), 42,06 kJ/mol (30 ${ }^{\circ}$ Brix) e 22,12 kJ/mol (50 ${ }^{\circ}$ Brix) e taxas de deformação de 0 a $400 \mathrm{~s}^{-1}$. Dak et al. (2006) também relataram para suco de manga Totapuri, entre temperaturas de 20 a $70{ }^{\circ} \mathrm{C}$, nas velocidades de rotação de 0,3 a 100 rpm e nas concentrações de 5,17, 8,51, 12,38 e 17\% de sólidos totais, valores de $E_{a}$ entre 1,66 e 11,35 kJ/mol, com a menor concentração de sólidos apresentando o maior valor de $E_{a}$. De acordo com Bezerra et al. (2009), os valores da energia de ativação indicam a sensibilidade da viscosidade aparente à alteração da temperatura, ou seja, altos valores de energia de ativação denotam o grau de influência da variação da temperatura sobre os valores de viscosidade. De acordo com Steffe (1996), a variação da temperatura pode ocorrer durante o processamento 

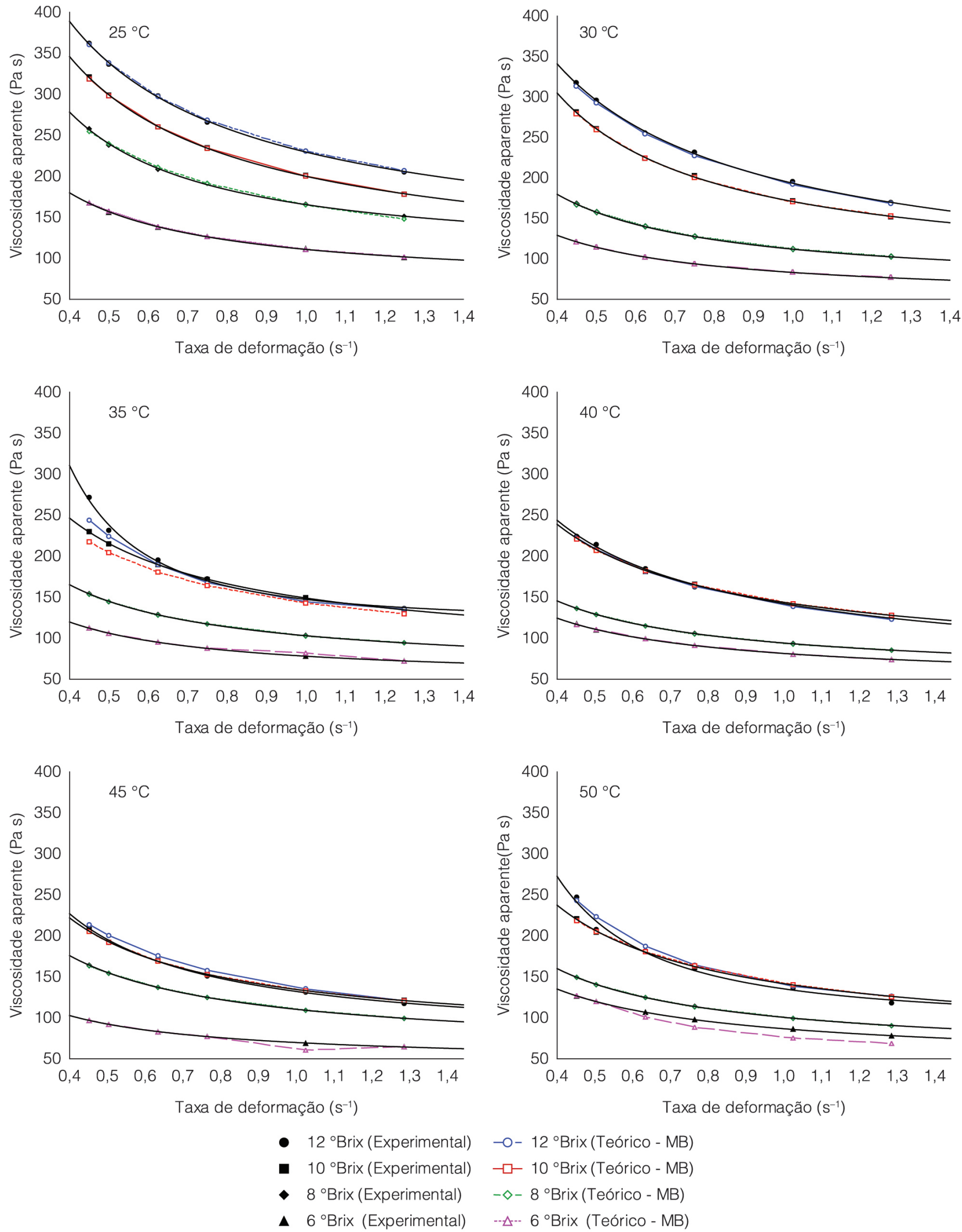

Figura 2. Relação da viscosidade aparente em função da taxa de deformação para a polpa de pequi com diferentes teores de sólidos solúveis totais, calculada com o modelo de Mizrahi-Berk. 
Tabela 5. Valores da energia de ativação $\left(E_{a}\right)$ da polpa de pequi em diferentes teores de sólidos solúveis totais na taxa de deformação de $1,25 \mathrm{~s}^{-1}$.

\begin{tabular}{|c|c|c|}
\hline $\begin{array}{c}\text { Sólidos solúveis } \\
\text { totais ( }{ }^{\circ} \text { Brix) }\end{array}$ & $\mathrm{Ea}\left(\mathrm{kJ} \mathrm{g}^{-1} \mathrm{~mol}^{-1}\right)$ & $\mathbf{R}^{2}$ \\
\hline 6 & 41,30 & 0,94 \\
\hline 8 & 39,09 & 0,94 \\
\hline 10 & 19,05 & 0,97 \\
\hline 12 & 27,21 & 0,96 \\
\hline
\end{tabular}

e o armazenamento dos alimentos, tendo influência nas suas propriedades reológicas.

Observaram-se em trabalhos com determinação de energia de ativação de polpa de frutas grandes variações nos resultados, com valores aproximados aos da polpa de pequi, como nos de Nindo et al. (2005), em sucos de mirtilo e framboesa em diferentes concentrações de sólidos solúveis totais (10 a $65^{\circ}$ Brix) e nas taxas de deformação entre 1 a $500 \mathrm{~s}^{-1}$, nos quais a $E_{a}$ do mirtilo chegou até 39,15 $\mathrm{KJ}$ mol-1 e a de framboesa, a $41,2 \mathrm{~kJ} / \mathrm{mol}$. Outros autores relatam em seu trabalho valores menores, como Gratão et al. (2007) para o suco de graviola com 9,3 a 49,4 Brix e nas velocidades de rotação de 0,028 a 243 rpm, trabalho no qual o valor máximo de $\mathrm{E}_{\mathrm{a}}$ atingiu 11,98 $\mathrm{kJ} \mathrm{g}^{-1} \mathrm{~mol}^{-1}$; e Guedes et al. (2010), que obtiveram para a polpa de melancia, com variação da taxa de deformação de 0 a $500 \mathrm{~s}^{-1}$ e em concentração de $8,17,26$ e $35^{\circ}$ Brix, $16,35 \mathrm{~kJ} \mathrm{~g}^{-1} \mathrm{~mol}^{-1}$ como maior valor.

\section{Conclusões}

A polpa concentrada apresentou comportamento pseudoplástico e os valores de viscosidade aparente apresentaram tendência de aumento com o aumento dos sólidos solúveis totais.

Os modelos de Ostwald-de-Waelle, HerschelBulkley e Mizrahi-Berk ajustaram-se bem aos dados reométricos, com melhores resultados para o de MizrahiBerk. A equação de Arrhenius na taxa de deformação estudada pode ser utilizada para expressar a influência da temperatura sobre a viscosidade aparente da polpa de pequi. A polpa de pequi tem características reológicas de natureza similar às de outras frutas e seu comportamento reológico, tanto sob variação de concentração como sob efeito do aquecimento, pode ser ajustado pelos mesmos modelos.

\section{Referências}

AHMED, J.; RAMASWAMY, H. S.; HIREMATH, N. The Effect of High Pressure Treatment on Rheological Characteristics and Colour of Mango Pulp. International Journal of Food Science and Technology, Oxford, v. 40, n. 1, p. 885-895, 2005. http:// $d x$. doi.org/10.1111/j.1365-2621.2005.01026.x
AUGUSTO, P. E. D.; IBARZ, A.; CRISTININI, M. Effect of High Pressure Homogenization (HPH) on the Rheological Properties of a Fruit Juice Serum Model. Journal of Food Engineering, Essex, v. 111, n. 2, P. 474-477, 2012. http://dx.doi.org/10.1016/j. jfoodeng.2012.02.033

ARÉVALO-PINEDO, A.; MACIEL, V. B. V; CARVALHO, K. M.; COELHO, A. F. S.; GIRALDO-ZUÑIGA, A. D.; ARÉVALO, Z. D. S.; ALVIM, T. C. Processamento e Estudo da Estabilidade de Pasta de Pequi (Caryocar brasiliense). Revista Ciência e Tecnologia de Alimentos, Campinas, v. 30, n. 3, p. 664-668, 2010. http:// dx.doi.org/10.1590/S0101-20612010000300015

BEZERRA, J. R. M. V.; RIGO, M.; DEMCZUK JUNIOR, B.; CÓRDOVA, K. R. V. Estudo do Efeito da Temperatura nas Propriedades Reológicas da Polpa de Morango (Fragaria ananassa). Revista Ambiência, Guarapuava, v. 5, n. 1, p. 37-47, 2009.

CHIN, N. L.; CHAN, S. M.; YUSOF, Y. A.; CHUAH, T. G.; TALIB, R. A. Modelling of Rheological Behaviour of Pumelo Juice Concentrates Using Master-curve. Journal of Food Engineering, Essex, v. 93, n. 1, p. 134-140, 2009. http://dx.doi. org/10.1016/j.jfoodeng.2009.01.005

DAK, M.; VERMA, R. C.; SHARMA, G. P. Flow Characteristics of Juice of "Totapuri" mangoes. Journal of Food Engineering, Essex, v. 76, n. 4, p. 557-561, 2006. http://dx.doi.org/10.1016/j. jfoodeng.2005.06.002

FERREIRA, G. M.; GUIMARÃES, M. J. O. C.; MAIA, M. C. A. Efeito da Temperatura e Taxa de Cisalhamento nas Propriedades de Escoamento da Polpa de Cupuaçu (T. grandiflorum Schum) integral. Revista Brasileira de Fruticultura, Jaboticabal, v. 30, n. 2, p. 385-389, 2008.

FERNANDES, T. K. S.; FIGUEIRÊDO, R. M. F.; QUEIROZ, A. J. M.; MELO, K. S.; BEZERRA, M. C. T. Estudo do Comportamento Reológico da Polpa de Umbu Cajá em Função da Concentração de Maltodextrina. Revista Brasileira de Produtos Agroindustriais, Campina Grande, v. 10, n. 2, p. 171-180, 2008.

GRATÃO, A. C. A.; SILVEIRA JUNIOR, V.; TELIS-ROMERO, J. Laminar Flow of Soursop Juice Through Concentric Annuli: Friction Factors and Rheology. Journal of Food Engineering, Essex, v. 78, n. 4, p. 1343-1354, 2007. http://dx.doi.org/10.1016/j. jfoodeng.2006.01.006

GUEDES, D. B.; RAMOS, A. M.; DINIZ, M. D. M. S. Efeito da Temperatura e da Concentração nas Propriedades Físicas da Polpa de Melancia. Brazilian Journal of Food Technology, Campinas, v. 13, n. 4, p. 279-285, 2010. http://dx.doi. org/10.4260/BJFT2010130400037

INSTITUTO ADOLFO LUTZ - IAL. Métodos Físico-químicos para Análise de Alimentos. São Paulo, 2008. 1020 p.

IZIDORO, D. R.; SCHEER, A. P.; SIERAKOWSKI, M. R.; HAMINIUK, C. W. I. Influence of Green Banana Pulp on 
Comportamento reológico e efeito da temperatura da polpa de pequi em diferentes concentrações SOUSA, E. P. et al.

the Rheological Behaviour and Chemical Characteristics of Emulsions (Mayonnaises). LWT - Food Science and Technology, Switzerland, v. 41, n. 6, p. 1018-1028, 2008.

NINDO, C. I.; TANG, J.; POWERS, J. R.; SINGH, P. Viscosity of Blueberry and Raspberry Juices for Processing Applications. Journal of Food Engineering, Essex, v. 69, n. 3, p. 343-350, 2005. http://dx.doi.org/10.1016/j.jfoodeng.2004.08.025

OLIVEIRA, M. E. B.; GUERRA, N. B.; BARROS, L. M.; ALVES, R. E. Aspectos Agronômicos e de Qualidade do Pequi. Fortaleza: Embrapa Agroindústria Tropical, 2008. 32 p. (Documentos, 113).

OLIVEIRA, R. C.; ROSSI, R. M.; BARROS, S. T. D. Estudo Reológico da Polpa de Morango (Fragaria vesca) em Diferentes Temperaturas. Acta Scientiarum Technology, Maringá, v. 34, n. 3, p. 283-288, 2012

OLIVEIRA, R. C.; ROSSI, R.; BARROS, S. T. D. Estudo do Efeito da Temperatura sobre o Comportamento Reológico das Polpas de Gabiroba e Goiaba. Acta Scientiarum Technology, Maringá, v. 33, n. 1, p. 31-37, 2011.

SATO, A. C. K; CUNHA, R. L. Influência da Temperatura no Comportamento Reológico da Polpa de Jabuticaba. Ciência e
Tecnologia de Alimentos, Campinas, v. 27, n. 4, p. 890-896, 2007. http://dx.doi.org/10.1590/S0101-20612007000400033

SHARMA, S. K.; LEMAGUER, M.; LIPTAY, A.; POYSA, V. Effect of Composition on the Rheological Properties of Tomato Thin Pulp. Food Research International, Oxford, v. 29, n. 2, p. 115-179, 1996.

STEFFE, J. F. Rheological Methods in Food Process Engineering. 2nd ed. Michigan: East Lansing, 1996. 418 p.

TONELI, J. T. C. L.; MURR, F. E. X.; PARK, K. J. Estudo da Reologia de Polissacarídeos Utilizados na Indústria de Alimentos. Revista Brasileira de Produtos Agroindustriais, Campina Grande, v. 7, n. 2, p. 181-204, 2005.

VIDAL, J. R. M. B.; SIERAKOWSKI, M. R.; HAMINIUK, C. W. I.; MASSON, M. L. Propriedades Reológicas da Polpa de Manga (Mangifera indica L. cv. Keitt) centrifugada. Ciência e Agrotecnologia, Lavras, v. 30, n. 5, p. 955-960, 2006.

VRIESMANN, L. C.; SILVEIRA, J. L. M.; PETKOWICZ, C. L. O. Chemical and Rheological Properties of a Starch-rich Fraction from the Pulp of the Fruit Cupuassu (Theobroma grandiflorum). Materials Science and Engineering C, Raleigh, v. 29, n. 2, p. 651-656, 2009. 